\title{
loT based Prototype for Smart Vehicle and Parking Management System
}

\author{
Garvit Pareek and M. Vinay \\ Department of Computer Science, Christ University, Bengaluru - 560029, Karnataka, India; \\ garvit.pareek@mca.christuniversity.in, vinay.m@christuniversity.in
}

\begin{abstract}
Objective: To formulate and simulate a constructive approach for decent parking space and vehicle safety using Internet of Things as both of them are of paramount importance in current scenario. Methods/Statistical Analysis: Internet of Things plays a vital role where everything is connected with everything. Flex sensors, switch, RFID and other stuff have been used to overcome the problem of vehicle parking. Already there have been early stage studies and has come up with prototype which shall aid user or drivers in finding the available parking space with the help of IOT. Findings: Since ample of related studies have been already done but integrating some new features can certainly pave the way of not only parking management but also in making the vehicle smart. For the purpose of parking facility, we check whether the slot is available, if so then the gate opens else not. Fee is charged on the time basis. Since the automobile industry and ICT industry amalgamate, the smart vehicle is a predominant issue. To make vehicle safe, we aim at fingerprint sensor. The fingerprint of every person is unique. The identification of an authorized user becomes more and more reliable. For the purpose of vehicle safety, we devise a fingerprint-based prototype along with GPS and GSM. The prime focus of the above prototype is to address traffic congestion, vehicle safety, and its current location. We also make sure using sensors integrated inside that the driver is not stoned and both the people sitting on the front row are tied up with seat belt. Applications/Improvements: The best part of this prototype is that it has been developed using RFID and IOT tool such as Arduino UNO, GPS, GSM and vibration motor. It not only enhances communication between the two or more connected devices but also maintains the transparency between them. All these can be achieved at cheaper rates.
\end{abstract}

Keywords: Parking System, Smart Vehicle, IOT, RFID

\section{Introduction}

In the current scenario, due to increase in traffic, ample of drivers roam around in search of decent parking space. This not only creates traffic congestion but also time waste. What if a driver has advance information of available parking space in the required destination spot. So using RFID, IOT we can plan and provide the parking slot to the driver in advance. For malls and airport, parking spaces are so extremely large that it is impossible to manage it manually. In urban areas like Bangalore, Delhi, Chennai, car parking has become a critical subject with the steady increase in count of vehicles. Research survey shows $40 \%$ of vehicles including car and bike in this traffic congestion are mere searching for a decent parking area. Survey also adds that on an average seven and a half minutes' time is needed to find a space for parking. A research says that $75 \%$ of devices in INDIA shall be connected to the internet by end of the year 2018. In this parking system, we set out IR sensors such a way that when a car is parked in a particular slot, an IR sensor will sense the presence of a vehicle in that slot and update the database. The server maintained here is of shared type. The data will be processed before sending to the database. People can easily book in advance the parking slot and accordingly, the shared database will be updated. Future of IOT can be seen where human interference is minimal, technology will take the control of everything.

${ }^{*}$ Author for correspondence 
Current automobile burglary cannot be just simply ignored. It is increasing day by day. Any person with a replica of original key can start the vehicle. The vehicle shall ignite irrespective of user. The user might drop the key or forget the key. It only adds frustration. Noticing the key point that anyone can start the vehicle and drive away, we coin a prototype that includes ARDUINO, fingerprint sensor, vibration motor, GPS, and GSM. Just like smartphones with thumb impression, the fingerprint scanner can be used to ignite the vehicle. Once the user has registered his thumb impression, he or she can use the vehicle. The vibration motor is to simulate the engine of a vehicle. Moreover, we can also add multiple users with different fingers. One the user start his bike, he shall also get message notification over his registered mobile number along with the current location. The location includes Longitude and latitude. Since multiple people can be registered, it makes easy for a family having a common vehicle among them. The reason behind using biometrics is that chances of getting into diplomacy are minimal. Whenever a user performs biometric authentication, the sensor takes the image of a thumb and determine the ridges and valley. It then matches the pattern with the previously scanned images.

\section{PROS}

- Difficult to fake.

- Nobody can guess the fingerprint just like a password.

- No one replaces or steal one's fingerprint.

- No need to remember anything.

Tracking down the theft vehicle is also a serious concern. If the colour of the vehicle and number plate is changed, it becomes more difficult for police to track down the thieves. RFID technology can be used to sort out this problem. RFID stands for Radio Frequency Identification. Since the name itself implies, it uses radio waves to identify the objects. These RFID can be fitted anywhere in the vehicle and be configured with any range of frequency. Using this RFID, we can trace the car details and its actual owner.

\section{Literature Survey}

"IoT based prototype for vehicle Parking Management System for Smart Cities" has proposed a framework for

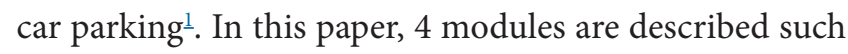
as book car slot online using any android app or web portal. Second, car entrance. If the slot is available then the gate will open else not. Third, it also checks for stolen or theft vehicle using RFID tag fitted inside the vehicle. Fourth, on exit gate fee will be charged based on a number of hours. These many factors in total contribute towards time-saving, power and unnecessary traffic.

"Minimization of Cruising for vehicle Parking" have implemented PMMS (Parking monitoring and management system) ${ }^{2}$. In this paper, data is collected regarding available slot and provide these details to the end user of PMMS's application. Information is also gathered from vehicle user, their smartphones, and the parking inspector. PMMS has 4 different parts - 2 mobile application, server, and database. The mobile application acts as an interface between the user and the database. Since there are two types of users - driver and parking inspector so there exist 2 applications. The database holds the information of available slots.

"Automatic vehicle Parking Management System and Fee Collection Based on Number Plate Recognition" have implemented an algorithm for the purpose of parking car automatically and collection of the fee ${ }^{3}$. In this micro-controller is pre-programmed so that there is no requirement of human intervention. OCR is used to identify and extract the details from number plate. Moreover, it also guides the user towards his parking slot. At exit gate, cash needs to be paid. It takes into account no. of hours car was parked by considering entry and exit time, the amount per hour, cash paid and change. If the user pays 10rs but they the fee amount was 7 then 3 Rs change must be given back to the user.

"An Intelligent Parking slot Management System" have implemented valet for smart parking at a low cost ${ }^{4}$. Fuzzy logic has been used for the above purpose. The vehicle will be provided with 2 modules - One is IR sensor and other is the microcontroller. IR sensor will detect any obstacle hindrance coming up in front so that user will not hit his vehicle. The IR sensor on the identification of obstacle at certain distance starts vibrating. Microcontroller will screen the parking slot closely and offer the necessary guidelines to the user. It is self-guided parking valet with minimal cost as there is no human interaction in this.

"An SMS Based Parking Reservation System", framework is proposed and implemented for smart parking using text message ${ }^{5}$. In this paper, the user needs to first 
get register with MCU service providers. When the user needs a slot, he/she sends a message requesting for the slot. GPS module incorporated with Arduino checks whether the message is authenticated or not. If not then no reply is given back. If the message is found true, then MCU with the help of WSN sends a message that includes parking slot number and a passcode. The user is supposed to enter the passcode at the entrance and exit of the parking area. Upon exit, the total fee is displayed on the LED screen.

"Car Safety System Enhancements using the Internet of Things (IoT)" have implemented a framework for people safety who is inside the car. The framework starts with alcohol detection ${ }^{6}$. If the driver is sober then car ignites else wait for $5 \mathrm{~min}$. If the alcohol is sensed during driving then it displays a message and car stops. The second framework is defined for other people sitting beside the driver. IR sensor is fitted next to seat buckle. If both buckles are ON then engine ignites else not. In case if the car meets with an accident, ARM sensor senses the car tilt and using GPS and GSM sends the message to the nearest police station and Ambulance along with the location.

"A Security and Safety measures based IoT Framework - embedded bikes" have proposed some improved solutions over existing framework in order to minimize bike and vehicle head-on collision ${ }^{2}$. The IoT based framework is integrated into bike and vehicle so that they can detect MDS and CPD. Misbehaviour Detection Scheme provides security awareness by sensing vehicle's mobility pattern based on misbehaviour detection. Collision Prediction and Detection scheme (CPD) keeps on monitoring driver's potential behaviour. Both the frameworks are an attempt to minimize the road accidents.

"Vehicle Alert and Collision Prevention System using Microcontroller" have proposed a hardware that includes one microcontroller, vibration sensor, GPS, EEPROM,

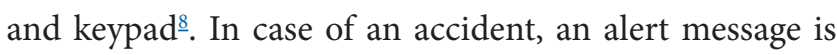
sent using GSM module to the people whose numbers is stored in EEPROM using keypad. The vibration sensor is responsible for the detection of an accident. The vibration sensor is connected to micro-controller using MCP. MCP is responsible for converting the analog signal into digital signal providing a digital output to the micro-controller. In addition, one LCD is also attached that shall display all the operation happening.

"An Intelligent System for Accident Detection and sending Notification" has proposed a framework in which using flex accelerometer sensor; accident can be easily detected ${ }^{2}$. Flex sensor is basically patented technology that identifies the accident accurately. The framework also includes a real-time camera along with GPS and GSM module. The sole purpose of this framework is to provide help to the people struck inside till the time ambulance or police arrive. GPS and GSM are used to send the alert message to police and hospital. A camera fitted inside will show the current condition of the people stuck inside.

"Wireless framework for Vehicle Accident Detection using Accelerometer sensor and Reporting with the help of GPS" has proposed a framework using microcontrollers to minimize death rates ${ }^{10}$. The framework includes Microcontroller, GPS, GSM, EEPROM, gas sensor, temperature sensor, alcohol sensor along with memory card and LED. Accelerometer sensor is used to detect the accident. Once the accident has happened, GPS will collect the current location of the accident and with the help of GSM will inform the nearest police station. The gas sensor is used to sense whether is there any leakage of gas, the temperature sensor will sense the temperature inside the vehicle, alcohol sensor is used to check whether the driver was sober or high. All the details will be stored in the memory card in order to identify the cause of the accident.

\section{Methodology}

The prototype we have coined is shown in Figure 1. The following prototype can be categorized into 4 parts:

(i) Book online (ii) Vehicle Entry (iii) Exit of the vehicle (iv) Parking Management.

The green colour board is basically a raspberry pi which will be our shared server. It is a low cost, smart card sized computing model that can be plugged into PC, laptop or TV. The entire coding is done in python and stored inside it. It uses an OS just like Linux and capable of performing any complex mathematical problem shown in Figure 2.

- Book Online: Since the drivers keep on roaming around seeking out for decent parking, using this feature they can book the space for car parking in advance. They just need to install the app and can select the vacant slot. For this feature, they don't have to pay anything in advance. Once booking is done, the server will be updated accordingly. Figure 3 shows the clear picture of how the online booking will be done in advance. It is same as booking a seat in the restaurant. 


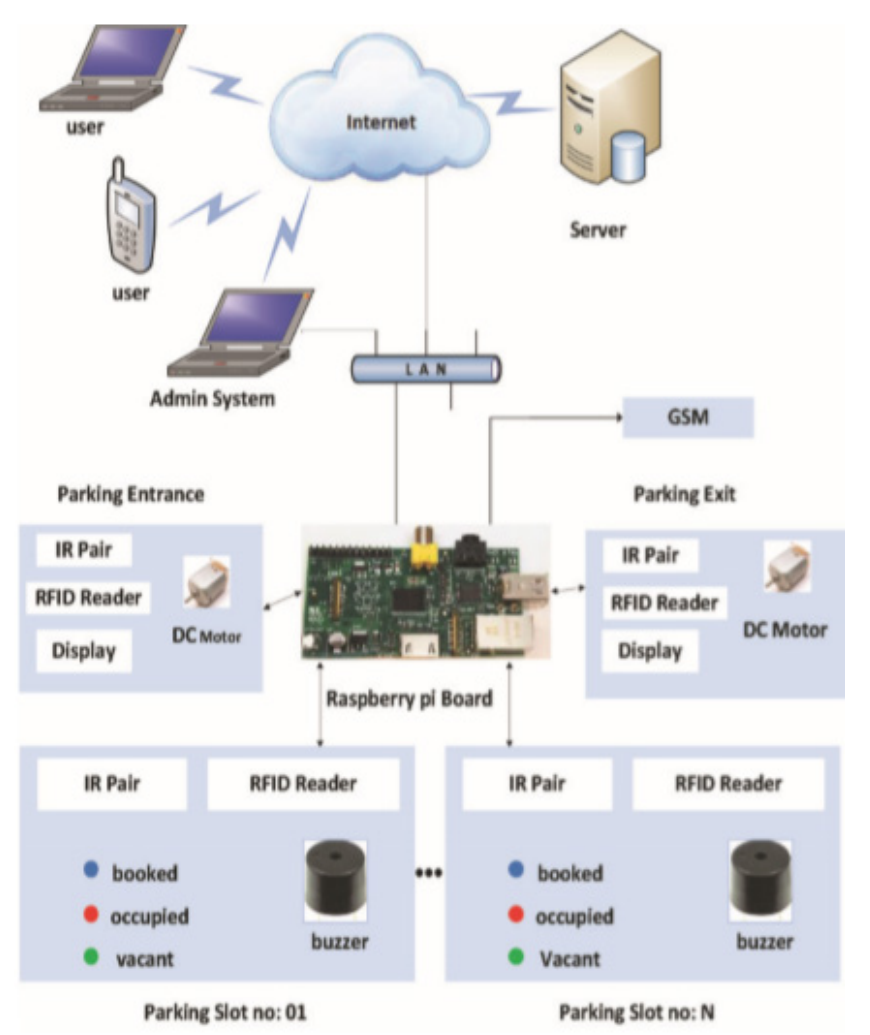

Figure 1. Prototype model for vehicle parking.

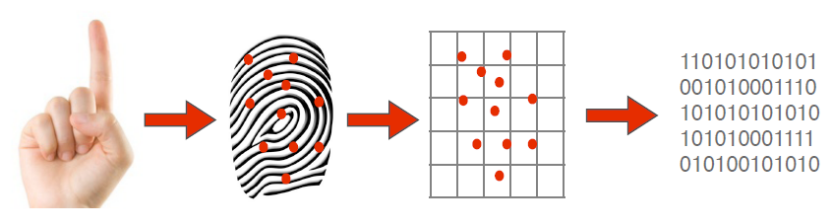

Figure 2. Mathematical Representation.

- Vehicle Entry: On the entrance of the parking gate, we need to check whether the car entering is stolen or not. If it so, then using IPV6 protocol, we will send an alert message to the closest police station. Internet uses a protocol suite called TCP/IP protocol. The purpose of this protocol is to identify host using the logical address and to route data over network layer of OSI model. Once the car enters, using RFID we cross-check the car details. If no issues, then the slot will be allocated based on availability. Figure 4 shows the clear picture of how vehicle entry will happen.

- Parking Management: Generally driver or user ends up in dilemma and parks the vehicle in the wrong slot. Not only this, incompetent drivers may hit other cars as

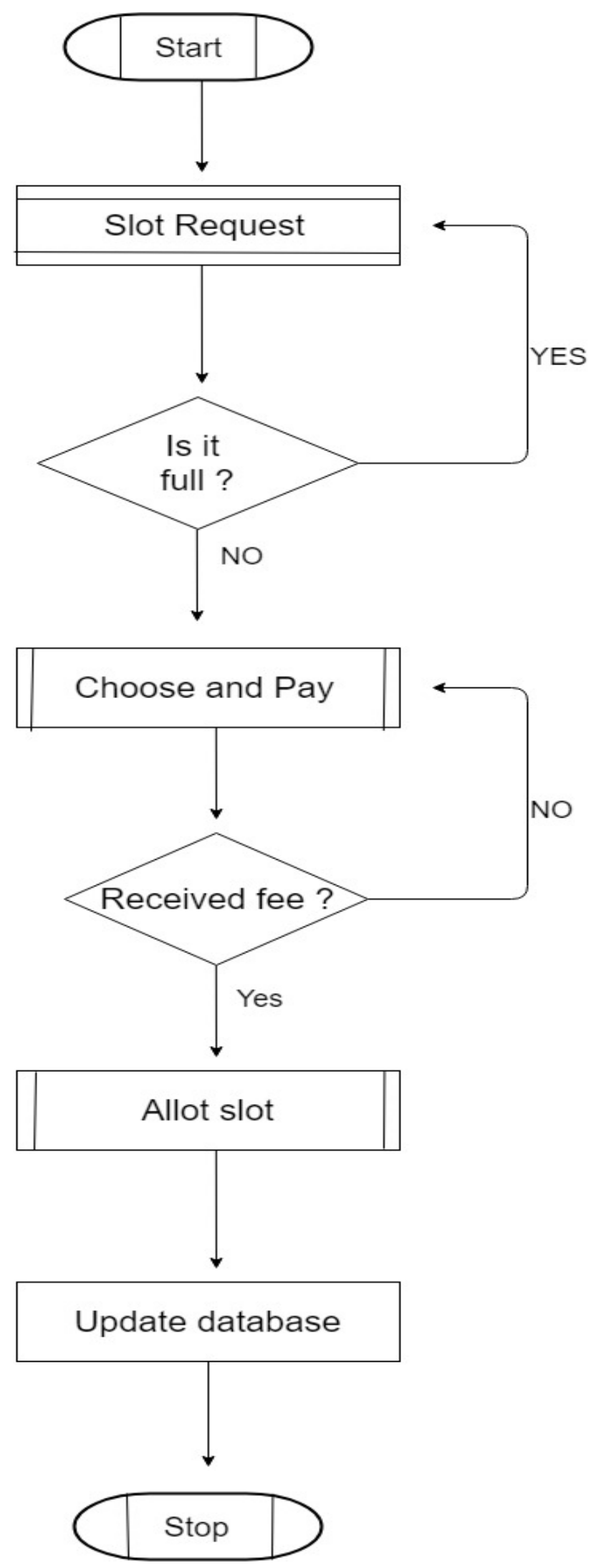

Figure 3. Flowchart for 'Book Online’. 


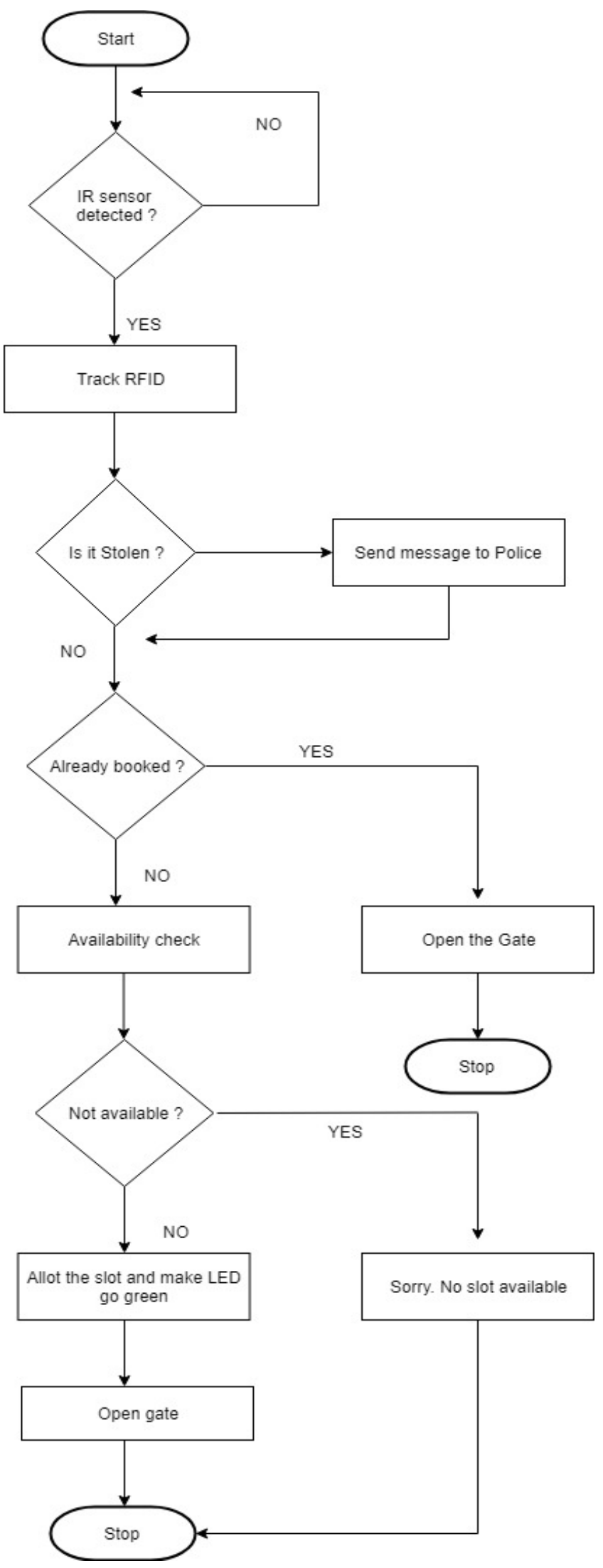

Figure 4. Flowchart for Vehicle Entry. well which causes frustration among people. The problem can be easily sorted out using the following algorithm which is demonstrated using flowchart in Figure 5.

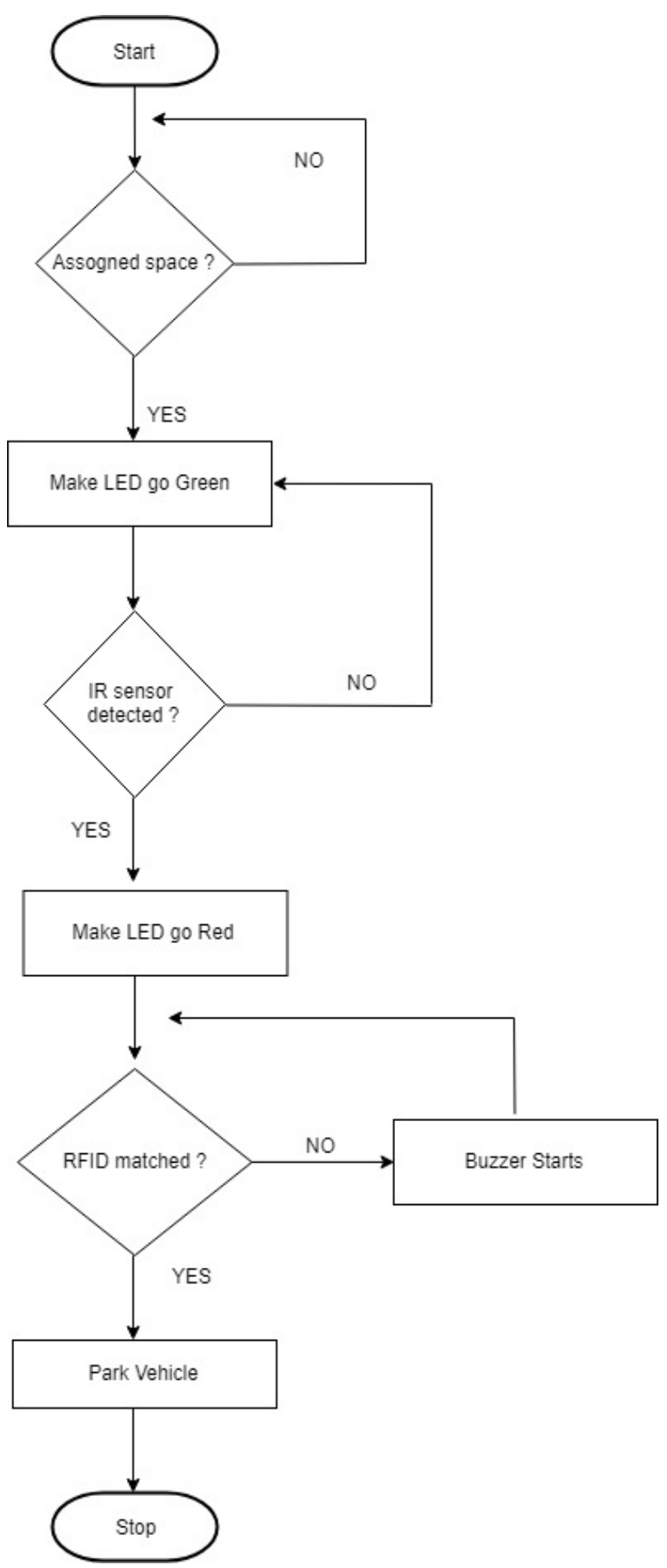

Figure 5. Parking Management Flowchart. 
- Exit of Vehicle: Upon leaving the parking space, the driver is required to pay the fee which will be calculated on time basis. Upon successful payment, that particular slot will make vacant again and the database will be updated accordingly so that next user can use the same. Figure 6 shows the entire flow of how the vehicle will exit,

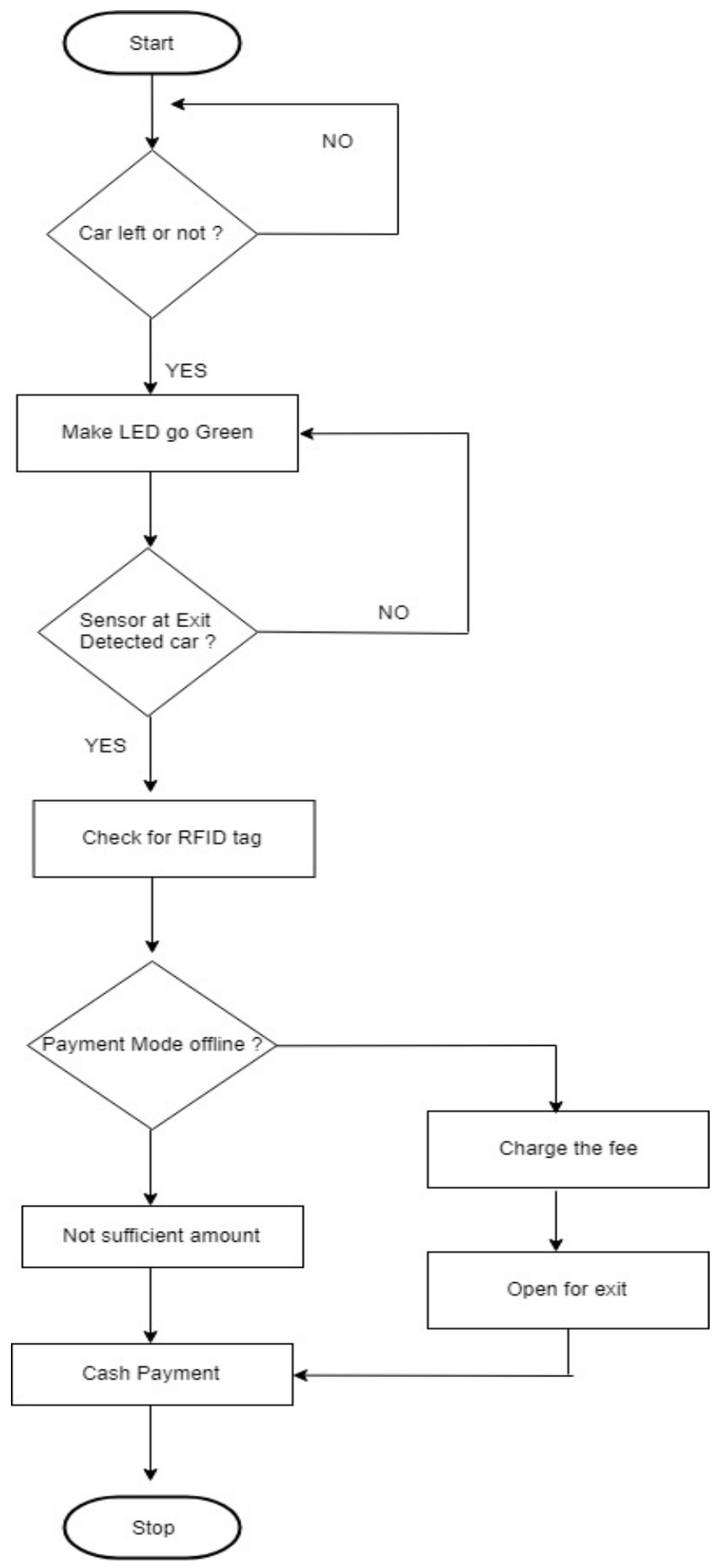

Figure 6. Flowchart of 'Exit of Vehicle'. how payment will happen and how slot shall be made available for next user.

Figure 7 given is the actual working model of Smart Parking System which is implemented using IoT technology using various sensors, switch, Arduino etc.

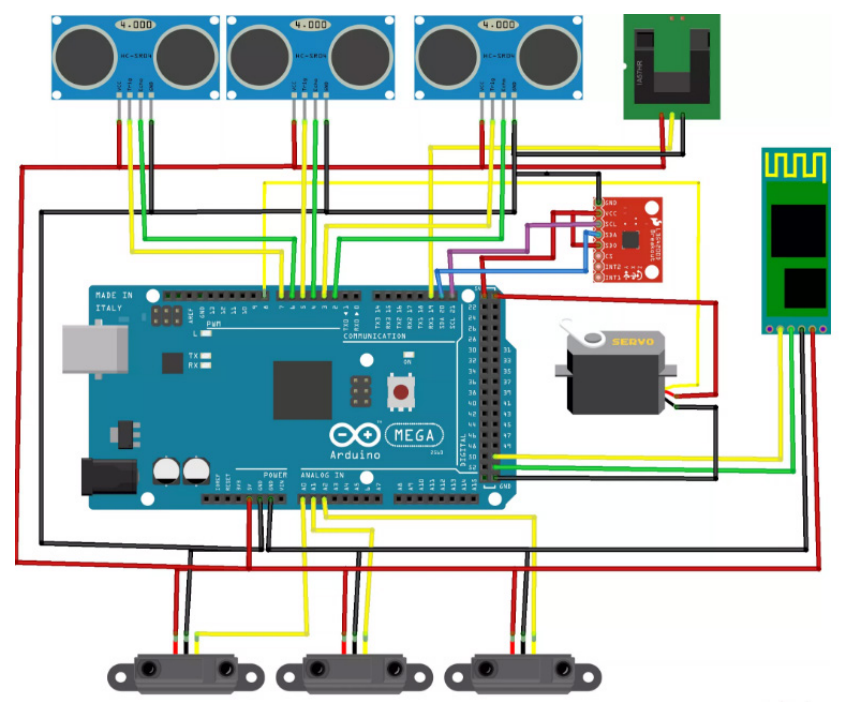

Figure 7. Working model for smart Parking.

So far we have seen how to find a decent parking space. But vehicle safety is another major challenge parallel to parking management. There is 3 module using that vehicle safety can be ensured.

Ardunio board encompasses different types of microprocessors and micro-controllers. It consists of numerous analog and digital input/output pins. The board is provided with Universal serial bus (USB) that can be used to attach to the laptop for loading the code. Arduino supports $\mathrm{C}$ and $\mathrm{C}++$ rules of code structuring.

The fingerprint sensor is attached to Arduino in order to scan the thumb impression. Basically, this sensor senses takes the image of thumb and check the ridges and valleys. If the user is authenticated then the motor starts else it sends a message to the user on his registered number saying that someone is trying to access your vehicle. Failing over 3 attempts, the message will be sent. The message will also include the latitude and longitude of the vehicle so that user can easily track his vehicle in case of emergency. Given Figure 8 is the flowchart of vehicle safety measure.

Global Packet Radio Service and Global System for Mobile Communication which is often shortened as GPRS and GSM are the modules that are used for commu- 


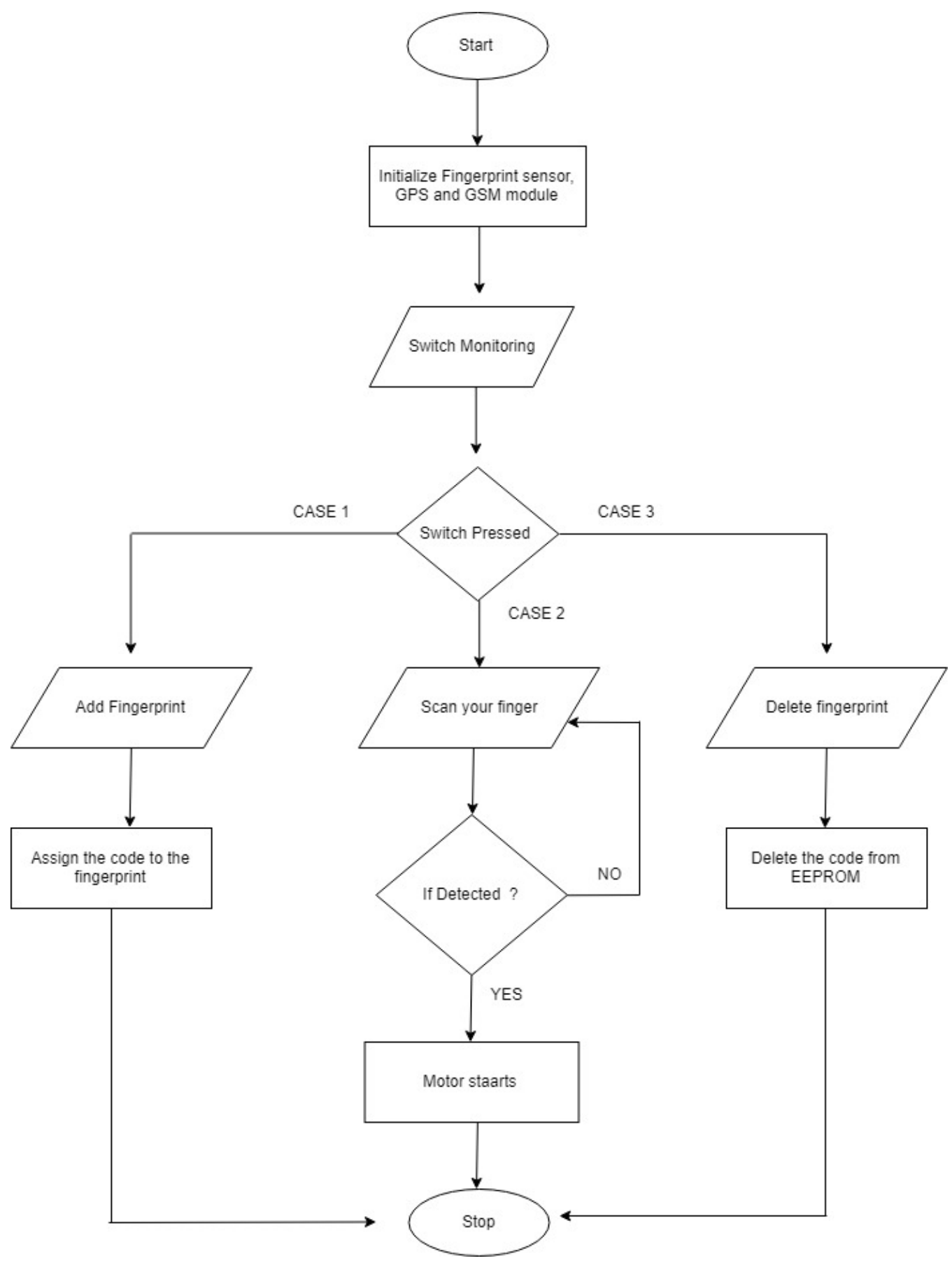

Figure 8. Flowchart for vehicle safety.

nication purpose. GSM module is a kind of modem that takes a SIM card as an input. Just like typical cell phone, it works over subscription plan. When this GSM module is attached to Arduino or raspberry pi, it allows them to get connected to the mobile operator. GPRS module basically creates and maintains the connection between
Arduino/raspberry and GSM module. GPS stands for Global Positioning System. It interacts with the satellite and calculates the ground location of a particular object. The GPS module used in our prototype is SIM900A which is cheap in cost and can be used to make calls. Send SMS and track the current location. 
Here arises one question. What if user's friend or family member wants to ride the vehicle? Technically not possible because only user's thumb is registered. The best part of this prototype is that more than one user can be registered at a single time so that multiple valid people can use the vehicle easily. The advantage of this prototype is that it's relatively very cheap and can be fixed irrespective of any vehicle. The following figures explain the flowchart of vehicle security.

\section{Conclusion and Further Scope}

In this research paper, using IoT technology, we have proposed a prototype that can easily be simulated in real time projects. The core objective of this paper is to contribute towards automobile industry using IOT technology. IOT support innovative ideas and provides tailored results. In the present scenario, everybody uses smart gadgets. IOT is just not a buzzword, its trending and can be used in any project. The above prototype provides the solution to traffic congestion and vehicle security. Not only this, stolen vehicles can also be tracked down easily. The user can easily park the vehicle within a fraction of seconds and can relax about its safety measures. There is a certain enhancement that can be made such as we can come up with the android application so that user can easily check and book the parking slot. We can even use IOT technology in safe driving by keeping an eye on the road. This way we can make an attempt to reduce the accidents.

\section{References}

1. Gandhi BMK, Kameswara RM. A Prototype for IoT based Car Parking Management system for Smart Cities. Indian Journal of Science and Technology. 2016; 9(17):1-6.
2. TsiarasC,HobiL,HofstetterF,LinigerS, StillerB.parkITsmart: Minimization of Cruising for Parking. 24th International Conference on Computer Communications and Networks (ICCCN). University of Zurich, Switzerland. 2015; p. 1-8. Crossref.

3. Rahman MA, Rashid MM, Farahana N, Musa A, Farhana A. Automatic vehicle Parking Management System and Fee Collection Based on Number Plate Recognition. International Journal of Machine Learning and Computing. 2012; 2(2):1-6.

4. Gebra S, Wang J, Wu Q, Sun Z, Zong K. IPLMS: An intelligent parking lot management system. Long Island Systems, Applications and Technology. 2015; p. 1-6.

5. Priyanka JGV, Hasnam M, Kumara WGCW. An SMS Based Parking Reservation System. 8th International Conference on Ubi-Media Computing; Colombo. 2015; 24-26:121-3.

6. Viral MV, Choksi V, Potdar MB. Car Safety System Enhancements using the Internet of Things (IoT). International Research Journal of Engineering and Technology (IRJET). 2017; 4(12):1-4.

7. Clarke S, Razzaque MA. Middleware for Internet of Things: A Survey. IEEE Internet of Things Journal. 2016; 3(1):70-95. Crossref.

8. Vardhini PH, Tushara DB. Wireless vehicle alert and collision prevention system design using Atmel microcontroller. IEEE International Conference on Electrical, Electronics, and Optimization Techniques (ICEEOT). 2016; p. 2784-7.

9. Vilas KA, Anil BS, Jagtap SR. Intelligent System for Accident Detection and sending Notification. IEEE International Conference on Communication and Signal Processing. 2014; p. 1238-40.

10. Bhavthankar S, Sayyed HG. Wireless framework for Vehicle Accident Detection using Accelerometer sensor and Reporting with the help of GPS. International Journal of Scientific \& Engineering Research. 2015; 6(8):1-4. 